\title{
RETRACTED ARTICLE: Correlated fracture precursors in rocks and cement based materials under stress
}

\author{
G. Niccolini · O. Borla • G. Lacidogna • \\ A. Carpinteri
}

Received: 20 May 2013/Accepted: 25 April 2014/Published online: 13 May 2014

(C) The Author(s) 2014

This article has been withdrawn by the Publisher and the Society in agreement with the Editor-in-Chief due to conflict of interest reasons. In a commitment to scientific integrity we decided to withdraw the article as the editorial process had been compromised.

G. Niccolini $(\bowtie) \cdot$ O. Borla · G. Lacidogna .

A. Carpinteri

Department of Structural, Geotechnical and Building

Engineering, Politecnico di Torino, C.so Duca degli

Abruzzi 24, 10129 Turin, Italy

e-mail: gianni_nicc@hotmail.com 